\title{
Occurrence and spatial distribution of antibiotic resistance genes in the Bohai Sea and Yellow Sea areas, China
}

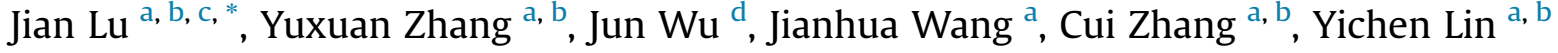 \\ ${ }^{a}$ CAS Key Laboratory of Coastal Environmental Processes and Ecological Remediation, Yantai Institute of Coastal Zone Research (YIC), Chinese Academy of \\ Sciences (CAS); Shandong Key Laboratory of Coastal Environmental Processes, YICCAS, Yantai Shandong, 264003, PR China \\ ${ }^{\mathrm{b}}$ University of Chinese Academy of Sciences, Beijing, 100049, PR China \\ ${ }^{c}$ Center for Ocean Mega-Science, Chinese Academy of Sciences, 7 Nanhai Road, Qingdao, 266071, PR China \\ d School of Resources and Environmental Engineering, Ludong University, Yantai, Shandong 264025, PR China
}

\section{A R T I C L E I N F O}

\section{Article history:}

Received 6 May 2019

Received in revised form

26 May 2019

Accepted 27 May 2019

Available online 28 May 2019

\section{Keywords:}

Antibiotic resistance genes

Bohai sea

Yellow sea

Coastal zone

Distribution coefficient

\begin{abstract}
A B S T R A C T
Rapid development of Bohai and Yellow Sea Economic Rim has led to the concern of emerging contamination of marine environments. This study investigated the spatial distribution of antibiotic resistance genes (ARGs) in Bohai and Yellow Sea areas. A large scale sampling from Bohai Sea, Yellow Sea and the major cities along the coastline from the mouth of Yalu River to the Yangtze River was performed. The spatial distribution of target ARGs based on the absolute abundances was in the trend of river water $\approx$ coastal water $>$ the Bohai Sea $>$ the Yellow Sea, inshore $>$ offshore and inner bay $>$ bay mouth. The total absolute abundances of selected ARGs in the coastal waters $\left(1.23 \times 10^{4}-3.94 \times 10^{5}\right.$ copies $/ \mathrm{mL}$ ) were about $1-4$ orders of magnitude higher than those in the sea $\left(21.1-8.00 \times 10^{3}\right.$ copies/ $\mathrm{mL}$ ). The abundances of ARGs fluctuated greatly in the Yellow Sea and the coastal areas. Sulfonamide resistance genes hold the highest abundances in the Bohai and Yellow Sea (up to $2.13 \times 10^{3}$ copies $/ \mathrm{mL}$ of sul 1 and $6.23 \times 10^{3}$ copies/mL of sul2), followed by tetracycline and quinolone resistance genes, while qnrA hold the highest abundances in coastal areas (up to $3.66 \times 10^{5}$ copies $/ \mathrm{mL}$ ). The distribution coefficients of target genes between sediments and corresponding water samples were more than 1.0 in the majority of different aquatic systems. According to the principle component analysis and redundancy analysis, water samples collected from the sea clustered together while those from the coastal zone and rivers were separated. Ammonium and nitrate played important roles in the distribution and variation of ARGs. Co-occurrence network analysis revealed that the potential multi-antibiotics resistant bacteria were detected with higher abundances in the Yellow Sea than in the Bohai Sea. These observations provided a comprehensive new insight into the pollution status of ARGs in the Bohai and Yellow Sea areas.
\end{abstract}

() 2019 Elsevier Ltd. All rights reserved.

\section{Introduction}

Antibiotics have been widely used to prevent and cure bacterial infections of animals and human beings, and promote animal growth in aquaculture and livestock farming (Lu et al., 2018). Meanwhile, a lot of antibiotics have been transported into natural environment via feces and urine by humans and animals,

\footnotetext{
* Corresponding author. CAS Key Laboratory of Coastal Environmental Processes and Ecological Remediation, Yantai Institute of Coastal Zone Research (YIC), Chinese Academy of Sciences (CAS); Shandong Key Laboratory of Coastal Environmental Processes, YICCAS, Yantai Shandong, 264003, PR China.

E-mail address: jlu@yic.ac.cn (J. Lu).
}

agricultural and livestock wastes, and discharge of wastewater treatment plants (WWTPs) (Lu et al., 2018). The total usage of antibiotics was approximately 162,000 tons in China in 2013, among which tetracyclines, sulfonamides, fluoroquinolones and macrolides were the dominant antibiotics, and animal consumption contributed to about 52\% (Lu et al., 2018; Zhang et al., 2015). The abuse and unmonitored discharge of antibiotics resulted in the occurrence, propagation and dissemination of antibiotic resistance genes (ARGs) and antibiotic resistant bacteria in receiving aquatic environment (Pruden, 2014). Regarded as emerging environmental pollutants, ARGs have become a public health concern to attract wide attention (Dang et al., 2017).

Nowadays, many studies have shown a high abundance of ARGs 
conferring resistance to tetracyclines, sulfonamides, quinolones and macrolides in water and sediments, especially in the aquatic environment. Occurrence and distribution of ARGs in various aquatic systems even including the drinking water treatment plant have been reported (Chen et al., 2019b; Luo et al., 2010; Wang et al., 2019). Five classes of ARGs in water and sediments from the Haihe River were quantified with the abundance ranging from $10^{4}$ to $10^{11}$ copies/g, and sulfonamide and tetracycline resistance was the most prevalent resistance (Dang et al., 2017; Luo et al., 2010). Additionally, ARGs are prevalent and proliferate in aquaculture environment with the introduction of antibiotics, which is a major anthropogenic threaten to the coastal water (Wang et al., 2019).

The Bohai Sea and Yellow Sea Economic Rim are industrialized and densely populated in recent decades, and the environment surrounding the sea has changed dramatically (Duan et al., 2010). According to monitoring data, land-based pollution contributed to about $80 \%$ of the total pollution with the major pollution sources including the discharge of industry, agriculture, animal husbandry, fishery, tourism, and so on. Rivers contributed to approximately $80 \%$ of pollutants such as heavy metals, ammonia, and phosphorus in the Bohai Sea and Yellow Sea (Song and Duan, 2019a, b). To the best of our knowledge, no comprehensive and systematical studies on investigating the distribution and abundances of ARGs in the Bohai Sea and Yellow Sea areas have been performed. It is important and indispensable to detect and quantify ARGs in the Bohai Sea and Yellow Sea areas since they are the important economic rim along the coastline of China. In this study, the abundances of class 1 integron-integrase gene (intI1) and ARGs belonging to four classes (sulfonamides, tetracyclines, quinolones and macrolides) were quantified. The objective of this study was to investigate the spatial distribution of target ARGs and intI1 in the Bohai Sea and Yellow Sea, the coastal zone and rivers, and various functional zones (mariculture, estuaries, tourist areas and ports). The correlations between the environmental factors, antibiotics and the abundances of selected ARGs were evaluated. The co-occurrence analysis between ARGs and microbial communities was also conducted. These data could provide a comprehensive and new insight on the pollution status of ARGs in the Bohai Sea and Yellow Sea areas.

\section{Materials and methods}

\subsection{Study area and sampling strategy}

A large-scale sampling from the Bohai Sea, the Yellow Sea and the major cities along the coastline from the mouth of Yalu River to the Yangtze River was performed in this study. Surface seawater samples and sediments in the Bohai Sea and Yellow sea were collected during voyage on the Chuang Xin I and Ke Xue III, respectively, from August 18 to September 7, 2018. Water samples and sediments along the seacoast and in the rivers were collected from July 17 to September 30, 2018. Coastal sampling sites were about $2-3 \mathrm{~m}$ off the coast, and river sampling sites were located in rivers which were $3-8 \mathrm{~km}$ to the estuaries. As shown in Fig. 1, 12 sampling sites were located in the Bohai Sea areas (BS), 9 sampling sites were located in the Yellow Sea (YS), 19 sampling sites were located along the coastline $(\mathrm{CZ})$, and 6 sampling sites were located in rivers (RV). Sampling locations covered the sea, coastal zone and rivers, and different functional areas such as the estuaries, tourist areas, maricultural zones and ports. The Yellow River, Liao River, Hai River, Xiaoqing River and Jie River deliver a considerable amount of freshwater and sediment to the Bohai Sea while the Yangtze River is the main river which discharges directly to the southern Yellow Sea. For this reason, river water samples were collected from these representative inflow rivers.

For samples from the sea, surface seawater was collected at
$50 \mathrm{~cm}$ depth into pre-cleaned plastic bottles, and surface sediments were collected using stainless steel box samplers. For samples from the coastal zone and rivers, surface water was collected by precleaned plastic bottles, and sediments were collected by sterile disposable syringe. Since surface water was more susceptive to various pollution, only surface water samples were collected during the investigation. At each sampling site, surface water samples with the volume of $30 \mathrm{~L}(5 \mathrm{~L} \times 6)$ and sediments with $600 \mathrm{~g}(100 \mathrm{~g} \times 6)$ were collected from six randomly chosen locations and mixed well, stored at cooler containers with ice boxes, and transported to laboratory immediately for further dispose and analysis.

\subsection{Physicochemical analysis}

Water samples were filtered through $0.45 \mu \mathrm{m}$ glass fiber membrane filters (Merck Millipore Ltd, Ireland) for the measurement of water quality parameters and antibiotics. The detailed experimental information referred to Lu et al. (2018). A total of seventeen antibiotics were analyzed, including four tetracyclines (tetracycline (TC), oxytetracycline (OTC), chlortetracycline (CTC), doxycycline (DOC)), eight sulfonamides (sulfamonomethoxine (SMM), sulfadiazine (SDZ), sulfamethazine (SMZ), sulfachinoxalin (SCX), sulfadimethoxine (SDM), sulfameter (SM), sulfaclozine (SCZ), sulfamethoxazole (SMX)), four quinolones (norfloxacin (NFC), ciprofloxacin (CFC), ofloxacin (OFC), enrofloxacin (EFC)) and one macrolide (roxithromycin (RTM)). Salinity was measured using a portable refractometer (LH-Y100, Lohand Biological, China) and a continuous flow analyzer (Auto Analyzer III, Seal, Germany)was utilized to measure the ammonia $\left(\mathrm{NH}_{4}^{-}-\mathrm{N}\right)$, nitrate $\left(\mathrm{NO}_{3}^{-}-\mathrm{N}\right)$, nitrite $\left(\mathrm{NO}_{2}^{-}-\mathrm{N}\right)$, reactive phosphate $\left(\mathrm{PO}_{4}^{3-}-\mathrm{P}\right)$ and reactive silicate $\left(\mathrm{SiO}_{3}^{2-}-\mathrm{Si}\right)$ of water samples.

\subsection{DNA extraction}

Water samples with a volume of $1.0 \mathrm{~L}$ filtered through $0.22 \mu \mathrm{m}$ mixed cellulose esters membranes (Millipore) and sediment samples $(0.5 \mathrm{~g})$ from each sampling site were used for the total DNA extraction. The sediments were lyophilized prior to DNA extraction. The total DNA was extracted by TIANamp Soil DNA Kit (TIANGEN Biotech, Beijing, China) according to the manufacturer's instructions. The concentration, purity and quality of extracted DNA were measured by a NanoDrop Lite UV-vis spectrophotometer (Thermo Scientific, Wilmington, USA) and the 1\% agarose gel electrophoresis.

\subsection{Quantification of ARGs, intI1 and $16 S$ rRNA gene}

The occurrence and absolute abundances of target ARGs, intI 1 and 16S rRNA gene in water samples and sediment samples were quantified by a Bio-Rad real-time (qPCR) system with 384-well plates (Bio-Rad CFX384 Touch, CA, USA). The target ARGs included sulfonamide resistance genes (sul1, sul2), tetracycline resistance genes (tet $\mathrm{B}$, tet $\mathrm{G}$, tet $\mathrm{X}$ ), macrolide resistance genes (erm $\mathrm{F}$, erm $\mathrm{T}$ ) and quinolone resistance genes (qnrA, qnrB, qnrS). And the selected ARGs could be divided into five different resistance mechanisms, such as antibiotic efflux (tetB, tetG), antibiotic inactivation (tetX), antibiotic target replacement (sul1, sul2), antibiotic target alteration (ermF, erm $\mathrm{T}$ ) and antibiotic target protection ( $q \mathrm{nrA}$, $q n r \mathrm{~B}, q n r \mathrm{~S})$. The detailed information of all primers used in this study for PCR amplification referred to Wang et al. (2019) and Zhu et al. (2017) and summarized in Table S1.

Each real-time PCR reaction was performed in $10.0 \mu \mathrm{L}$ system, including 5.0 $\mu \mathrm{L} \mathrm{SYBR}^{\circledR}$ Premix Ex Taq ${ }^{\mathrm{TM}}$ II (Takara, Dalian, China), 1.0 $\mu \mathrm{L}$ DNA template, $0.4 \mu \mathrm{L}$ forward primer, $0.4 \mu \mathrm{L}$ reverse primer $(0.4 \mu \mathrm{M})$ and $3.2 \mu \mathrm{L} \mathrm{ddH}_{2} \mathrm{O}$. Each reaction was run in triplicate for 


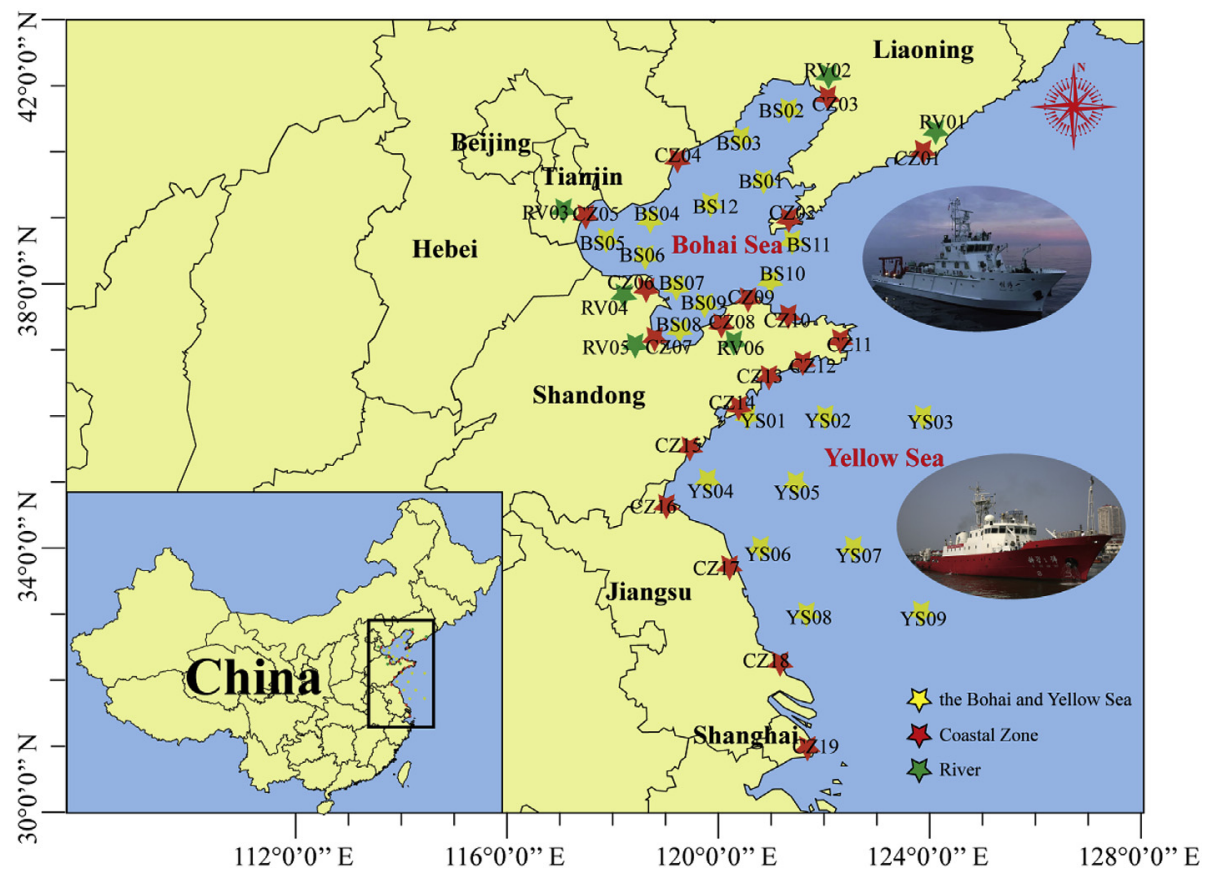

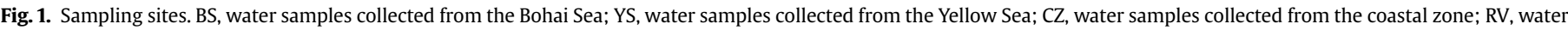
samples collected from rivers. (For interpretation of the references to color in this figure legend, the reader is referred to the Web version of this article.)

each water and sediment DNA samples and sterile $\mathrm{ddH}_{2} \mathrm{O}$ was used as negative control. Real-time PCR amplified reactions were run with the following program: pre-denaturation at $95^{\circ} \mathrm{C}$ for $30 \mathrm{~s}, 40$ cycles of denaturation at $95^{\circ} \mathrm{C}$ for $5 \mathrm{~s}$, and annealing for $30 \mathrm{~s}$ at annealing temperature. Finally, a melting curve was carried out with temperature ranging from $60^{\circ} \mathrm{C}$ to $95^{\circ} \mathrm{C}$. Besides, gene of qnrA was performed with three-steps method, 40 cycles of denaturation at $95^{\circ} \mathrm{C}$ for $5 \mathrm{~s}$, annealing for $30 \mathrm{~s}$ and extension at $72^{\circ} \mathrm{C}$ for $40 \mathrm{~s}$. The absolute abundances of target genes were quantified based on calibration standard curves which were set up using ten-fold dilution serial of plasmids DNA ranging from $10^{0}$ to $10^{7}$ copies $/ \mu \mathrm{L}$. The plasmids DNA was extracted from $E$. coli DH5 $\alpha$ cloned by the PMD19-T vector (TaKaRa), which was ligated with PCR products of target genes. Quantification of target genes was considered to be effective with features including the melting curves with a single peak, the amplification efficiencies in the range of $90 \%-110 \%$ and the $\mathrm{R}^{2}$ values of calibration standard curves higher than 0.99 .

The relative abundance of target ARGs and intI 1 were calculated as the followings:

Relative abundance $=$ Copies of target gene/Copies of 16S rRNA gene

In order to figure out whether the ARGs tend to be accumulated in the sediment or water phase, the distribution coefficient $(K d)$ of target genes between the water phase and sediment phase was calculated by the following equation:

$K d=A s / A w$

As represents the absolute abundance of target gene in the sediment, and $A w$ represents the corresponding gene absolute abundance in the surface water.

\subsection{Illumina MiSeq sequencing}

The purified total DNA extracted from water and sediment samples was sent to Majorbio (Shanghai, China) for Illumina MiSeq sequencing (Illumina, San Diego, USA). The V4-V5 hypervariable regions of the bacterial 16S rRNA genes were amplified by thermocycler PCR system (GeneAmp 9700, ABI, USA), with pairs of primers 515F (5'-GTGCCAGCMGCCGCGG-3') and 907R (5'CCGTCAATTCM TTTRAGTTT $-3^{\prime}$ ). The PCR amplification and the PE300 paired-end sequencing was carried out according to the standard procedures of Majorbio. Raw sequencing data were quality-filtered and merged by Trimmomatic and FLASH, OTUs (operational taxonomic units) with 97\% similarity were clustered using UPARSE (version 7.1), and chimeric sequences were removed by UCHIME. Each $16 \mathrm{~S}$ rRNA gene sequence was classified and analyzed by RDP classifier algorithm against the Silva database. The raw reads of all samples were deposited into the National Center for Biotechnology Information (NCBI) database (Accession Number: SRP194150).

\subsection{Statistical analysis}

Statistical analysis of the abundances of target genes was plotted by Origin (Version 2019, Origin Lab Corporation, USA). Pearson's correlation analysis was conducted using SPSS 19 (IBM, USA). Principal component analysis (PCA) was accomplished by Origin. Redundancy analysis (RDA) was conducted by Canoco 5.0. Network analysis between detected genes and bacteria was performed in $\mathrm{R}$ environment with vegan and igraph packages and further visualized by Cytoscape 3.7.1.

\section{Results and discussion}

\subsection{Distribution and abundances of ARGs in different aquatic systems}

As shown in Fig. 2A, the total abundances of the target ARGs ranged from $2.05 \times 10^{2}$ copies $/ \mathrm{mL}$ to $7.25 \times 10^{3}$ copies $/ \mathrm{mL}$ in the Bohai Sea and varied greatly from 21.1 copies $/ \mathrm{mL}$ to $8.00 \times 10^{3}$ 


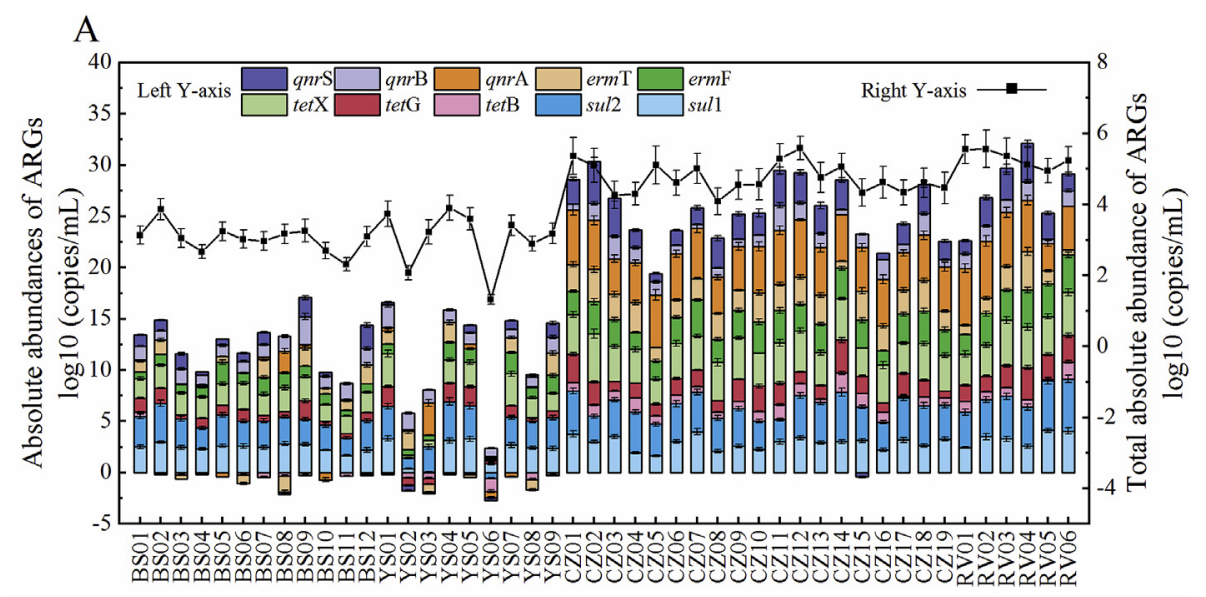

B

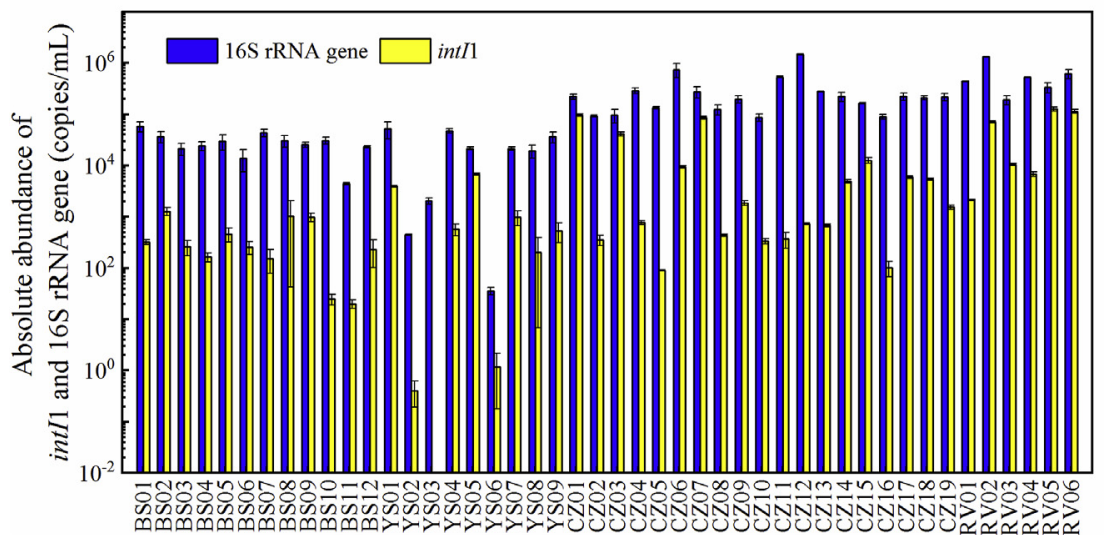

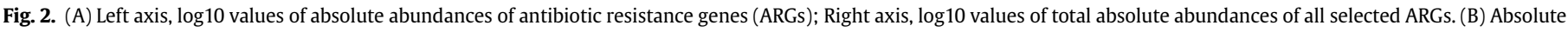
abundance (copies/mL) of $16 \mathrm{~S}$ rRNA gene and class 1 integron-integrase gene (intI1).

copies/mL in the Yellow sea. In coastal areas, the abundances of ARGs were obviously higher than those in the Bohai Sea and Yellow Sea, ranging from $1.23 \times 10^{4}$ copies $/ \mathrm{mL}$ to $3.94 \times 10^{5}$ copies $/ \mathrm{mL}$ in coastal water and from $8.91 \times 10^{4}$ copies/mL to $3.67 \times 10^{5}$ copies/ $\mathrm{mL}$ in rivers. The overall trend of the concentrations of target ARGs was river water $(\mathrm{RV}) \approx$ coastal water $(\mathrm{CZ})>$ the Bohai Sea $(\mathrm{BS})>$ the Yellow Sea (YS). This result indicated that the coastal zone was more susceptible to the land-based pollution, thereby holding higher abundances of ARGs than the sea. Meanwhile, large discharge of sediments and freshwater with land-based contaminant from more than forty rivers flowed into the Bohai Sea, resulting in the accumulation of ARGs in the Bohai Sea.

In the Bohai Sea, Liaodong Bay (BS01, BS02 and BS03), Bohai Bay (BS04, BS05 and BS06), Laizhou Bay (BS07, BS08 and BS09), Bohai Strait (BS10 and BS11) and the central sea basin (BS12) were selected as the objects to investigate the spatial distribution of ARGs. The total absolute abundances of selected ARGs in the inner bay were higher than those in the bay mouth, such as BS02 > BS01 \& BS03 and BS05 > BS04 \& BS06. Interestingly, the total absolute abundances of selected ARGs decreased sharply in the Bohai Strait (BS10 and BS11), but increased in the central sea basin (BS12). The spatial distribution features of ARGs might be caused by the geographic features of the Bohai Sea. The Bohai Sea is a semienclosed continental shelf sea in China with $80 \%$ of the pollutants originating from land sources (Song and Duan, 2019a). Besides, the low concentration of ARGs in the Bohai Strait was owing to the relatively stronger currents. Higher concentration in the central sea basin might be influenced by the mixing and diffusion of landbased and marine-based pollutants. As for the target ARGs, sulfonamide resistance genes (sul1 and sul2) hold the highest abundances in the range of 45.6 copies $/ \mathrm{mL}$ (BS11) - $9.55 \times 10^{2}$ copies $/ \mathrm{mL}$ (BS02) and 54.0 copies/mL (BS11) - $6.04 \times 10^{3}$ copies $/ \mathrm{mL}$ (BS02), respectively. BS02 was located near the mouth of the Daliaohe River in the Liaodong bay, which was an important industrial base in Liaoning province. A lot of aquaculture and animal husbandry farming, industries, chemical and medicine factories are located and distributed in these coastal cities (Lu et al., 2015). TetX with the abundances ranging from 53.4 copies/mL to 342.3 copies $/ \mathrm{mL}$ was rich and ubiquitous in all the twelve seawater samples, and followed by qnrB, ermF, tet $\mathrm{G}$ and qnrS. Other ARGs (tetB, erm $\mathrm{T}$ and qnrA) were detected with low abundances and great variation in different sampling sites. Gene qnrA was detected with low absolute abundances in all sites while it was relatively rich ( 137.6 copies $/ \mathrm{mL}$ ) in BS08 which was located in the Laizhou Bay with about ten inflow rivers such as the Xiaoqinghe, Huanghe and Weihe Rivers.

In the case of the Yellow Sea, the absolute abundances of ARGs decreased from the inshore areas to the offshore areas, such as YS01 > YS02 and YS04 > YS05, and the abundances of the detected ARGs in YS02, YS03 and YS06 were relatively low. The quantification result showed that the absolute abundances of different ARGs fluctuated greatly in different sampling sites in the Yellow Sea. It might be owing to the great quantity of complex freshwater and sediments discharge from land. There are nearly thirty rivers flow into the Yellow Sea (Song and Duan, 2019b). Among the target 
ARGs, the absolute abundances of sul genes (sul1 and sul2) were the highest, up to $2.13 \times 10^{3}$ copies $/ \mathrm{mL}$ (sul 1 ) and $6.23 \times 10^{3}$ copies $/ \mathrm{mL}$ (sul2), respectively. Specially, the abundance of sul1 in YSO2 and YSO3 was much lower than that in YS01, with the decreasing trend from inshore to offshore. TetX showed relatively higher absolute abundances in water samples than tet $\mathrm{B}$ and tet $\mathrm{G}$ owing to the different antibiotic resistance mechanisms, which was similar to previous reports (Chen et al., 2019b). The resistance mechanism of tetB and tetG was antibiotic efflux and that of tetX was antibiotic inactivation. Interestingly, qnrB showed higher abundances in all samples while qnrA and qnrS showed relatively lower abundances and fluctuated widely. Gene qnrA was rich in YSO3 with the absolute abundance of $1.35 \times 10^{3}$ copies $/ \mathrm{mL}$. This result might be attributed to the special geography, complicated circulation patterns and the abundant supply derived from the Yangtze River, the Yellow River and rivers in Korea (Song and Duan, 2019b).

The total absolute abundances of ARGs in the coastal water samples collected along the coastline were about 1-4 orders of magnitude higher than those detected in the Bohai and the Yellow Sea. It has been reported that the target ARGs (sul, tet and qnr etc.) were detected in water from the Pearl River Delta region with variable abundances ranging from $1.75 \times 10^{2}$ copies/mL to $4.00 \times 10^{6}$ copies/mL (Su et al., 2017). The absolute abundance of qnrA was the highest in the coastal water samples, ranging from $7.56 \times 10^{3}$ copies $/ \mathrm{mL}$ to $3.66 \times 10^{5}$ copies $/ \mathrm{mL}$, while $q n r \mathrm{~B}$ and $q n r \mathrm{~S}$ were detected with lower absolute abundances $(q n r \mathrm{~B} \leq 281.3$ copies/mL and $q n r S \leq 1.34 \times 10^{4}$ copies/mL). It has been hypothesized that qnrA emerged because of the selective pressure and the heavy use of quinolones in hospital and medicine industry, or acquired by the strains containing integrons (Cambau et al., 2006). Sulfonamide resistance genes (sul1 and sul2) were frequent and abundant in selected coastal water samples for the widespread use of sulfonamide antibiotics in aquaculture and graziery, but it had relatively lower abundances in the tourist areas and ports such as CZ02, CZ04, CZ05, CZ10 and CZ16. In contrast to qnrA, sul1 and sul2 persisted in the aquatic environment from the coastal areas to the sea with relatively higher abundances. For tetracycline resistance genes, tetX hold the highest absolute abundances in coastal water, up to $5.05 \times 10^{4}$ copies $/ \mathrm{mL}$. However, the abundances of tetB and tet $\mathrm{G}$ were low (tet $\mathrm{B} \leq 80.9$ copies/mL and tet $\mathrm{G} \leq 1.46 \times 10^{3}$ copies/ $\mathrm{mL}$ ). For macrolides resistance genes, the absolute abundances of erm $\mathrm{F}$ ranged from 25.9 copies/mL to $3.43 \times 10^{3}$ copies $/ \mathrm{mL}$, and the absolute abundances of erm T ranged from 4.76 copies $/ \mathrm{mL}$ to $1.42 \times 10^{3}$ copies $/ \mathrm{mL}$. In rivers, the absolute abundances of different ARGs were at similar level with those in the coastal water. Gene qnrA hold the highest average absolute abundances, followed by sul2, sul1 and tetX. The absolute abundances of the other target ARGs were relatively low and fluctuated widely.

Water samples in the river, the following estuary and the surrounding sea area were selected to obtain further information on the distribution of detected ARGs. In contrast, the absolute abundances of target ARGs topped at the river, followed by the following estuary and decreased 1-3 orders of magnitude in the surrounding sea area by taking the examples of RV01 $>$ CZ01, RV02 > CZ03 > BS02, RV03 > CZ05 > BS05, RV04>CZ06 > BS07, $\mathrm{RV05} \approx \mathrm{CZ07}>\mathrm{BS08}$ and $\mathrm{RV06}>\mathrm{CZ08}>\mathrm{BS09}$. This result was consistent with the previous investigations in the Taizhou Bay (Chen et al., 2019b). IntI1 played important roles in the acquisition and dissemination of antibiotic resistance genes among bacteria (Subirats et al., 2018). As demonstrated in Fig. 2B, intI1 was rich in the coastal water and river water with the absolute abundances up to $9.57 \times 10^{4}$ copies $/ \mathrm{mL}(\mathrm{CZ})$ and $1.27 \times 10^{5}$ copies $/ \mathrm{mL}(\mathrm{RV})$, respectively. On the contrary, the absolute abundances of intI 1 in the Bohai Sea and the Yellow Sea were at lower levels with the range of $10-10^{3}$ copies/mL and those at YS02, YS03 and YS06 were less than 1.18 copies/mL. Remarkably, intI1 was more abundant in the estuaries (such as CZ01, CZ03 and CZ07) with the abundances at the similar order of magnitude with those in the rivers, reflecting the great potential of transferability and environmental risks of ARGs in the coastal areas, especially in the estuaries.

\subsection{Relative abundances of ARGs in different sampling sites}

As shown in Fig. 2B, the absolute abundances of 16S rRNA genes

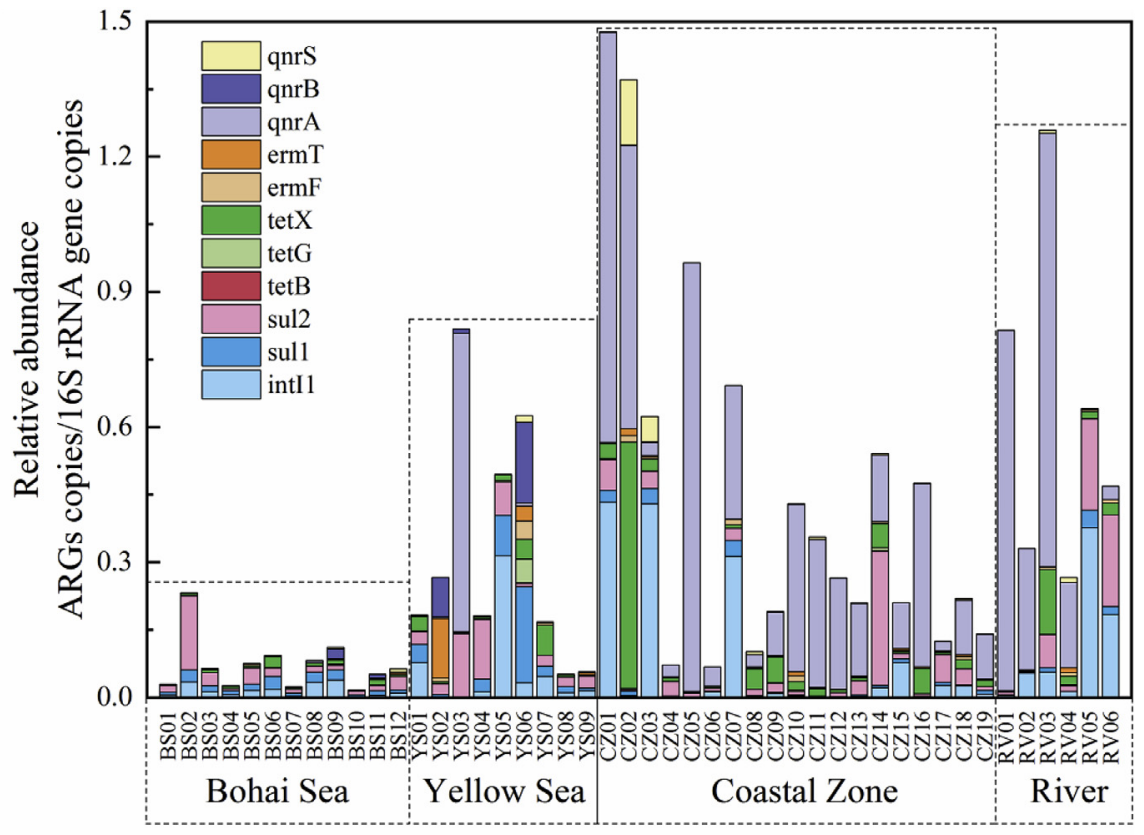

Fig. 3. Relative abundances (ARGs copies/16S rRNA gene copies) of antibiotic resistance genes (ARGs) and class 1 integron-integrase gene (intI1). 
were quantified with the range of $35.7-1.49 \times 10^{6}$ copies $/ \mathrm{mL}$ in all water samples. The absolute abundances of $16 \mathrm{~S}$ rRNA genes detected in the coastal water and river water samples were higher than those in seawater collected from the Bohai and Yellow Sea. For eliminating the influence of biomass, the copy numbers of ARGs were normalized to the copy numbers of the 16S rRNA gene (Liu et al., 2019). The relative abundances of target ARGs were higher in the coastal water and river water samples but lower in the sea, which was consistent with distribution of ARGs based on the absolute abundances (Fig. 3). The relative abundances of detected ARGs and intI1 were at low level and stable in the Bohai Sea while those were at high level and fluctuated considerably in the Yellow Sea and the coastal water.

The relative abundances of qnrA were the highest among all target ARGs. The relative abundances of qnrA mainly ranged from $10^{-3}$ to 0.4 and those of several samples (CZ01, CZ03 and RV03) were more than 0.9 . Genes including $q n r B$ and $q n r S$ were detected with low relative abundances less than 0.18 and 0.15 , respectively. The relative abundance of sul 1 ranged from $10^{-4}$ to $10^{-1}$ (most of abundance less than 0.05) and followed the trend of $\mathrm{YS}>\mathrm{BS}>\mathrm{RV}>\mathrm{CZ}$. In comparison to sul1, sul2 was detected with higher relative abundance and varied considerably with the range of $10^{-4}-10^{-1}$. This result further confirmed that sul genes persisted in the aquatic environment from the coast to the sea with high abundances. Similar result was obtained in the Bohai Bay (Zhang et al., 2018). The abundances of sulfonamide resistance genes were higher than those of the other ARGs, which might be attributed to the wide application of sulfonamide antibiotics in aquaculture system (Gao et al., 2018). For tetracycline resistance genes, tetX was detected at high relative abundance with the highest abundance up to 0.55 . However, the relative abundances of tetB and tet $\mathrm{G}$ were the lowest (less than 0.01 ) in the majority of water samples. Moreover, the macrolide resistance genes (erm) were detected at lower relative abundances, and the majority of them were less than 0.05 gene copies/16S rRNA gene copies. Notably, the relative abundances of intI 1 were lower than those of qnrA but higher than those of the other target ARGs. The spatial variation of ARGs and intI 1 based on the relative abundance was corresponding with that of target genes based on the absolute abundances.

\subsection{Relationships between ARGs, intI1 and antibiotics}

Only nine among seventeen target antibiotics were detected in coastal water and they included TC, OTC, CTC, DOC, SMX, SMM, NFC, CFC and RTM (Fig. S1). OTC, NFC, CFC and RTM were detected with high frequency (100\%), followed by TC, CTC and DOC (94.7\%). In contrast, SMX and SMM were detected with low frequency of $10.5 \%$. Concentrations of the dominant antibiotics were in the range of

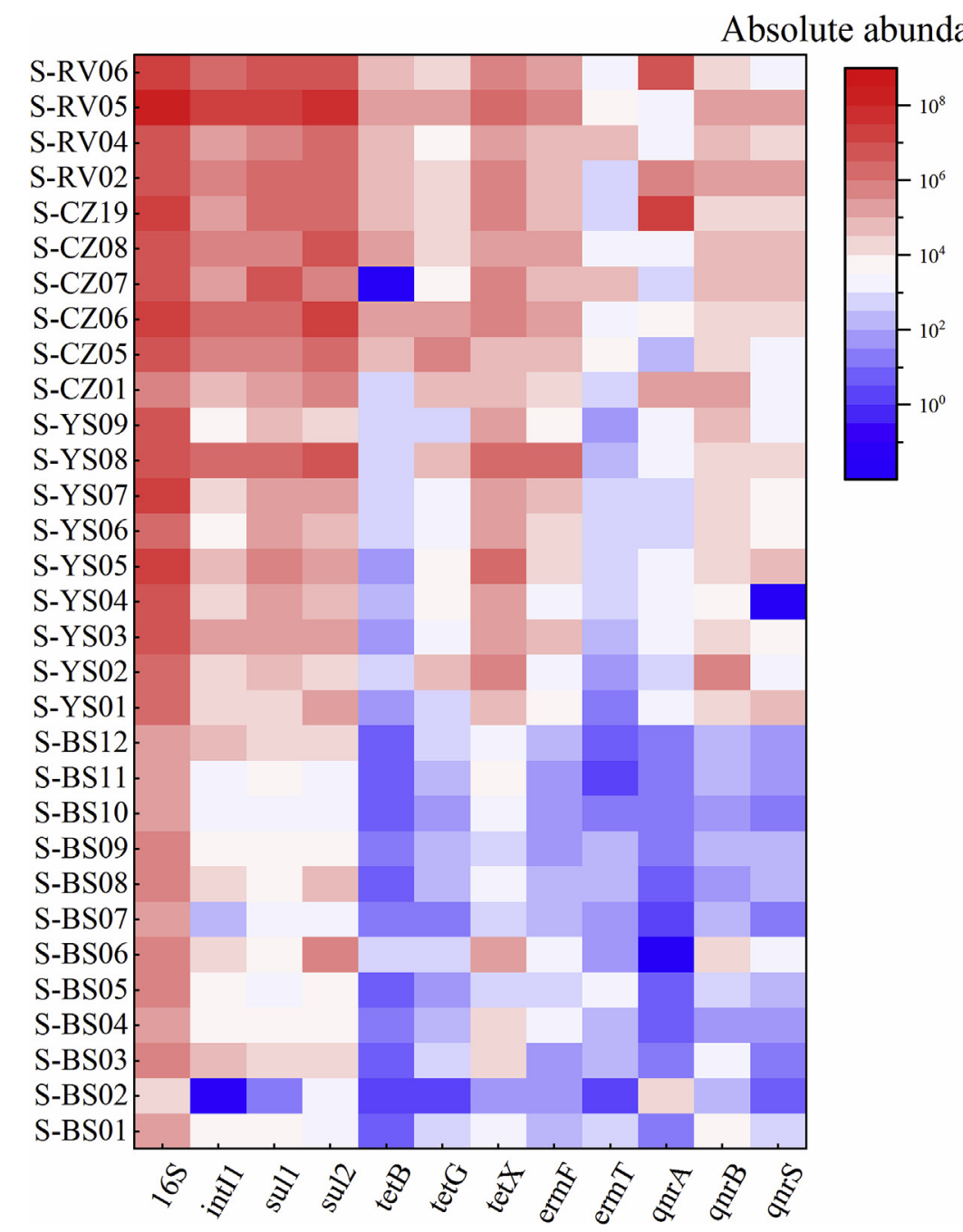

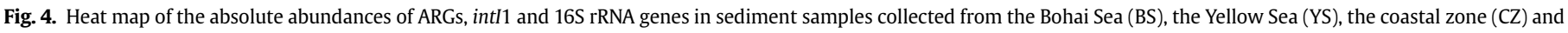
rivers (RV). (For interpretation of the references to color in this figure legend, the reader is referred to the Web version of this article.) 
373-384 ng/L (No detection in CZ15) for TC, 301-1070 ng/L for NFC and 243-255 ng/L for CFC, respectively. Besides, the concentrations of OTC, CTC, DOC and RTM were mainly in the range of 50-100 ng/ L.

Antibiotics played important roles in facilitating the enrichment and propagation of antibiotic resistance genes as a result of selection and fitness of antibiotic resistant bacteria (Zhang et al., 2018). Pearson's correlation coefficients between antibiotics, ARGs, intI1 and 16S rRNA gene were calculated and listed in Table S2. There were significant and positive correlations $(\rho>0.6, p<0.05)$ between intI1 \& sul1, sul2 \& tetG, sul2 \&tetX, tetG \& tetX, TC \& DOC, OTC \& NFC, OTC \&CFC, and SM \& SMM. The result indicated that there were positive and significant correlations between antibiotic \& antibiotic or ARG \& ARG. However, there was not direct correlation between antibiotics and the corresponding ARGs. Previous investigation has also shown that there was not direct correlation between antibiotics and the corresponding ARGs in coastal aquaculture systems (Wang et al., 2019).

\subsection{Distribution coefficients of ARGs in water samples and sediments}

As shown in Fig. 4, the absolute abundances of selected ARGs in sediments collected from the Bohai Sea $\left(4.67 \times 10^{3}-5.41 \times 10^{5}\right.$ copies/g) were significantly lower than those in sediments collected from the Yellow Sea $\left(3.88 \times 10^{5}-1.08 \times 10^{7}\right.$ copies $\left./ g\right)$, the coastal zone $\left(1.49 \times 10^{6}-1.74 \times 10^{7}\right.$ copies $\left./ g\right)$ and rivers $\left(1.98 \times 10^{6}-6.55 \times 10^{7}\right.$ copies/g). The abundances of $i n t I 1$ gene and sulfonamides resistance genes (sul1 and sul2) were high in these sediment samples, with the maximums up to $2.99 \times 10^{7}$ copies/g (intI1), $0.76 \times 10^{7}$ copies/g (sul1) and $4.50 \times 10^{7}$ copies/g (sul2), respectively. Similarly, the absolute abundances of sul1 and sul2 detected in the sediments from the East Sea of China were in the range of $10^{5}-10^{8}$ copies/g (Chen et al., 2019b). Notably, the spatial variation of detected ARGs and intI 1 in sediments was consistent with that in water samples, with the order of $C Z$ \& RV > BS \& YS. However, the absolute abundances of detected ARGs and intI 1 in sediments collected from the Bohai Sea were lower than those from the Yellow Sea, which was not consistent with the order of water samples owing to the sediment characteristics. This result might be attributed to that the environment in coastal areas was more likely to be influenced by the land-based pollution. Meanwhile, the surface sediments from the southern Yellow Sea was mainly supplied by the Yellow River and the Yangtze River, which consisted of mudflat, saltmarsh, silt or sand flat (Song and Duan, 2019b).

The distribution coefficient $(K d)$ was an important tool to reveal the distribution behaviors of emerging contaminants including ARGs in water and sediments. The distribution coefficients of target genes (ARGs, intI1 and 16S rRNA gene) in different aquatic environment (the Bohai Sea, the Yellow Sea, the coastal zone and rivers)
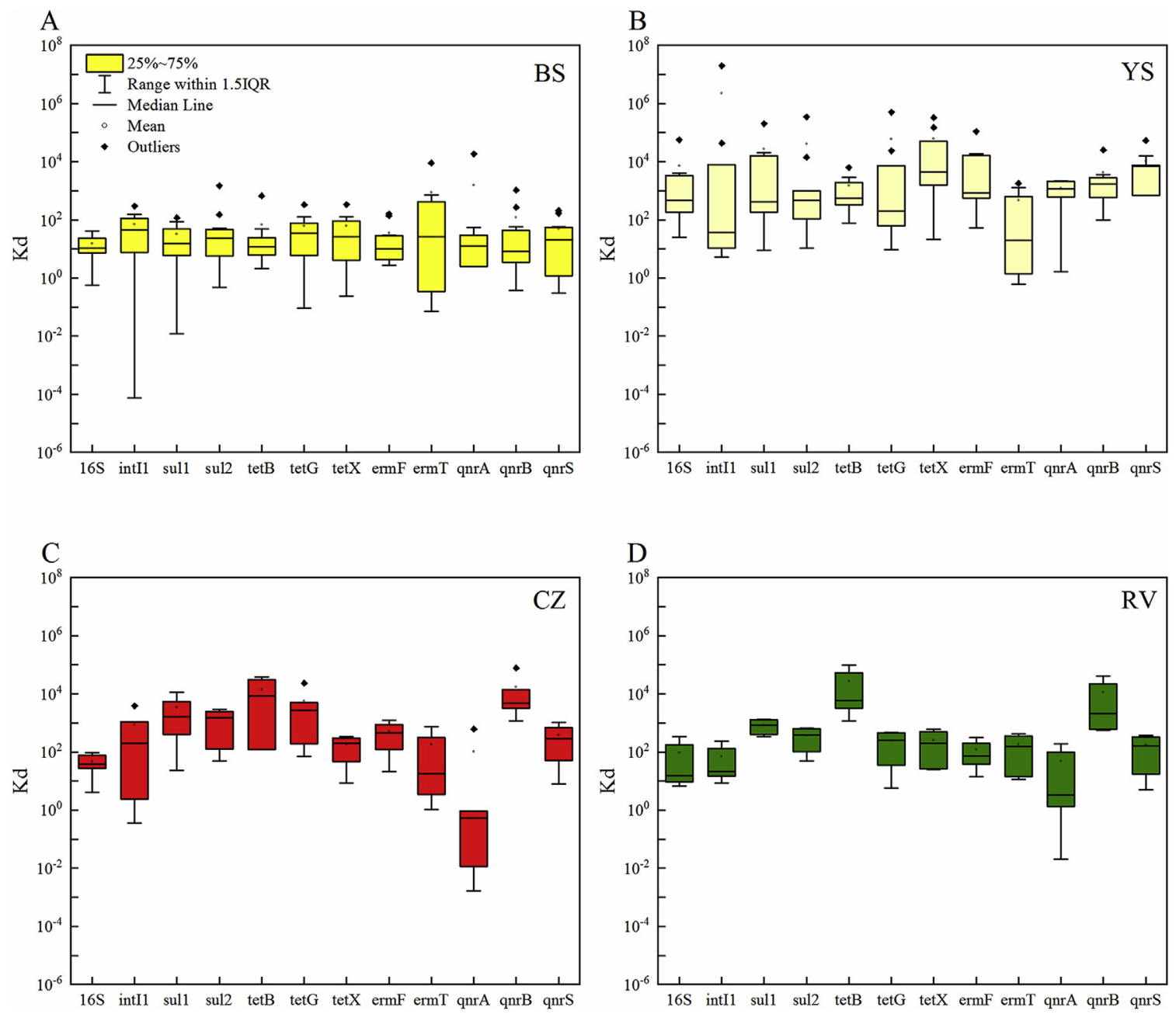

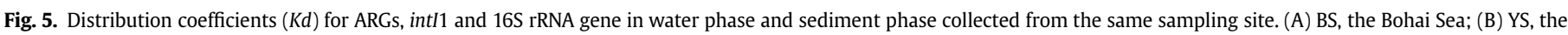
Yellow Sea; (C) CZ, coastal zone; (D) RV, rivers. (For interpretation of the references to color in this figure legend, the reader is referred to the Web version of this article.) 
were calculated and visualized by box plots (Fig. 5). The $K d$ values were generally more than 1.0 in the majority of different aquatic systems, indicating that ARGs were inclined to be accumulated in sediments. Based on these observations, sediments might serve as important reservoirs of ARGs in marine environments. It was worth noting that the $K d$ values of ARGs in the Yellow Sea were much higher than those in the Bohai Sea, and the $K d$ values of ARGs were highly variable in the coastal aquatic systems. The highest value of $K d\left(5.05 \times 10^{5}\right)$ was observed for tetG in the Yellow Sea area, indicating tet might be more readily accumulated in the sediment than other ARGs. The mean $K d$ value for tet $\mathrm{G}$ and tetX was $1.85 \times 10^{4}$ and $1.80 \times 10^{4}$, respectively. The properties and composition of the sediments in the Yellow sea (Song and Duan, 2019b) might lead to the relative high $K d$ values of ARGs. Meanwhile, the hypervariable $K d$ values of ARGs in the surface water-sediment systems in the coastal zones and rivers were owing to the complicated and volatile environment in coastal areas.

\subsection{Correlations between ARGs, intI1 and environmental factors}

Based on the absolute abundances of ARGs, intI1 and 16S rRNA gene, principal components analysis (PCA) was utilized to reveal the correlations and differences between different water samples (Ma et al., 2019). As shown in Fig. 6A and B, PC1 explained 34.2\% of the total target genes variation, and PC2 explained $18.7 \%$ of the variation. The samples collected from the Bohai Sea and Yellow Sea clustered together while the water samples from the coastal zone and rivers were separated. The result indicated that the variation of water samples from the coastal zone and river basin was notable, whereas the abundances of target genes in the sea were relatively stable. Owing to the complicate environment, the abundances of target ARGs, intI1 and 16S rRNA gene varied significantly among different water samples collected from the coastal zone and rivers, indicating that the coastal zone was more susceptible to the discharge and pollution from land than the sea.
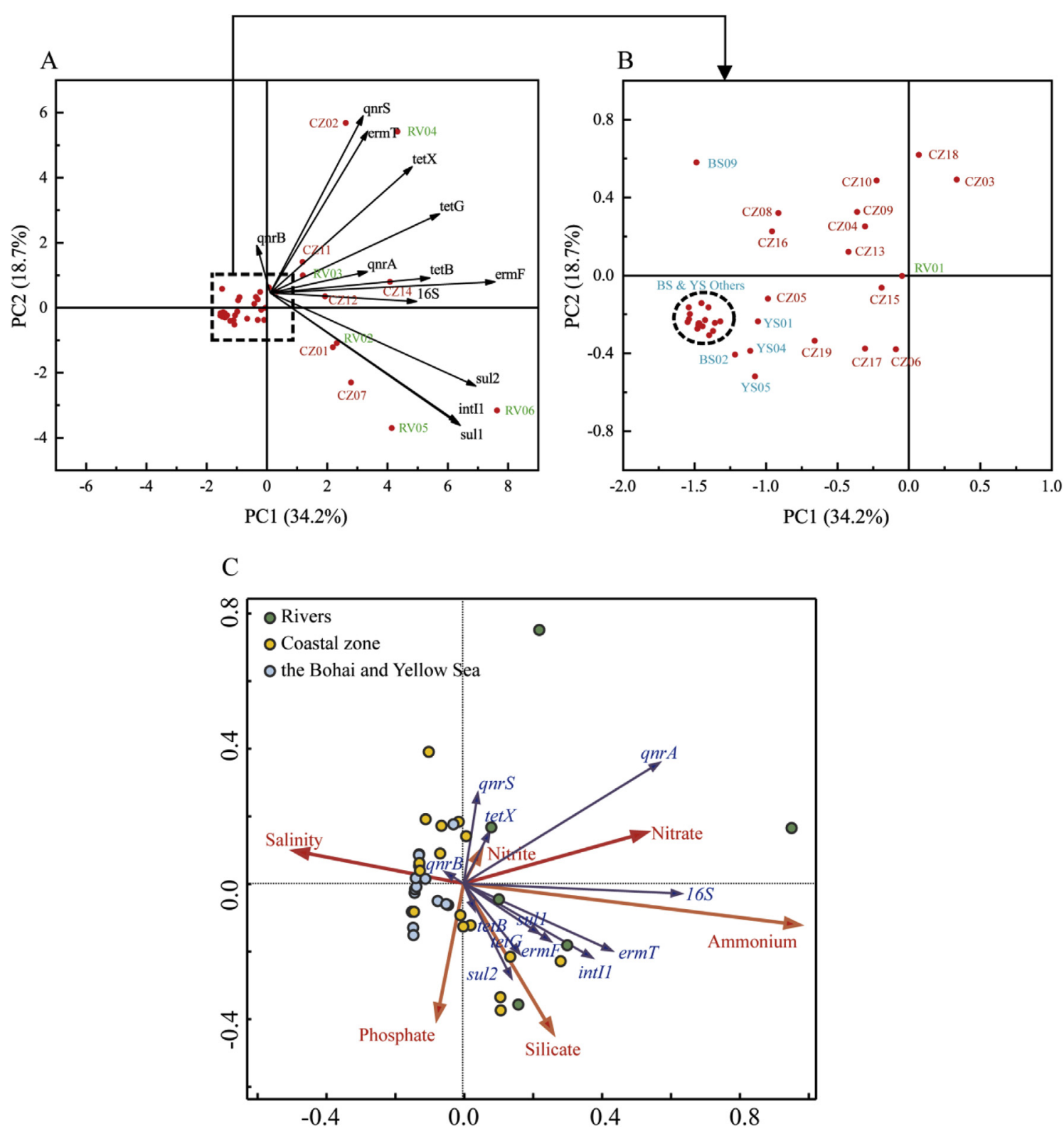

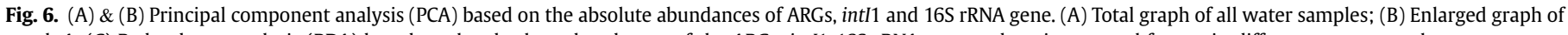
graph A. (C) Redundancy analysis (RDA) based on the absolute abundances of the ARGs, intI1, 16S rRNA gene and environmental factors in different water samples. 
Previous studies reported that the abundances and distribution of ARGs had close relationships with some environmental parameters (Di Cesare et al., 2016). Subsequently, redundancy analysis (RDA) was used to evaluate the relationships between target genes (ARGs, intI1, 16S rRNA gene) and the environmental factors including salinity, ammonium, nitrate, nitrite, reactive phosphate and silicate. As shown in Fig. 6C, ammonium $(p<0.01)$ and nitrate $(\mathrm{p}<0.05)$ played important roles in the distribution and variation of target genes. Among them, 16S rRNA gene, qnrA, ermF, ermT, sul1, sul2, tetG and intI1 showed higher positive correlations with ammonium and nitrate. As the main contamination index in aquatic environment, the concentration of ammonium should be paid attention closely. The composition of different water samples was demonstrated by the distance between the dots, and the correlations between water samples and the environmental factors were demonstrated by the projections onto the lines of environmental variables by the sample symbols (Chen et al., 2019a). The composition of river water samples (RV) was mainly influenced by the concentration of ammonium and nitrate, and the composition of coastal water was influenced by ammonium, nitrate and salinity. The clustering result of different water samples based on the RDA was consistent with that based on the PCA, further confirming that the water samples from rivers and the coastal zone were more susceptible to contamination.

\subsection{Co-occurrence patterns among ARGs, intI1 and bacterial communities}

A total of 1,508,847 optimized bacterial sequences were obtained from 21 water samples collected from the Bohai Sea and Yellow Sea. Proteobacteria, Cyanobacteria, Bacteroidetes, Actinobacteria and Firmicutes were the dominant bacterial phyla in the Bohai Sea and Yellow Sea (Fig. S2). Synechococcus, Candidatus_Pelagibacter, Exiguobacterium, Candidatus_Actinomarina, OM60_NOR5_clade, NS5_marine_group, NS4_marine_group, Alteromonas and Pseudoalteromonas were the dominant bacterial genera with the averages of relative

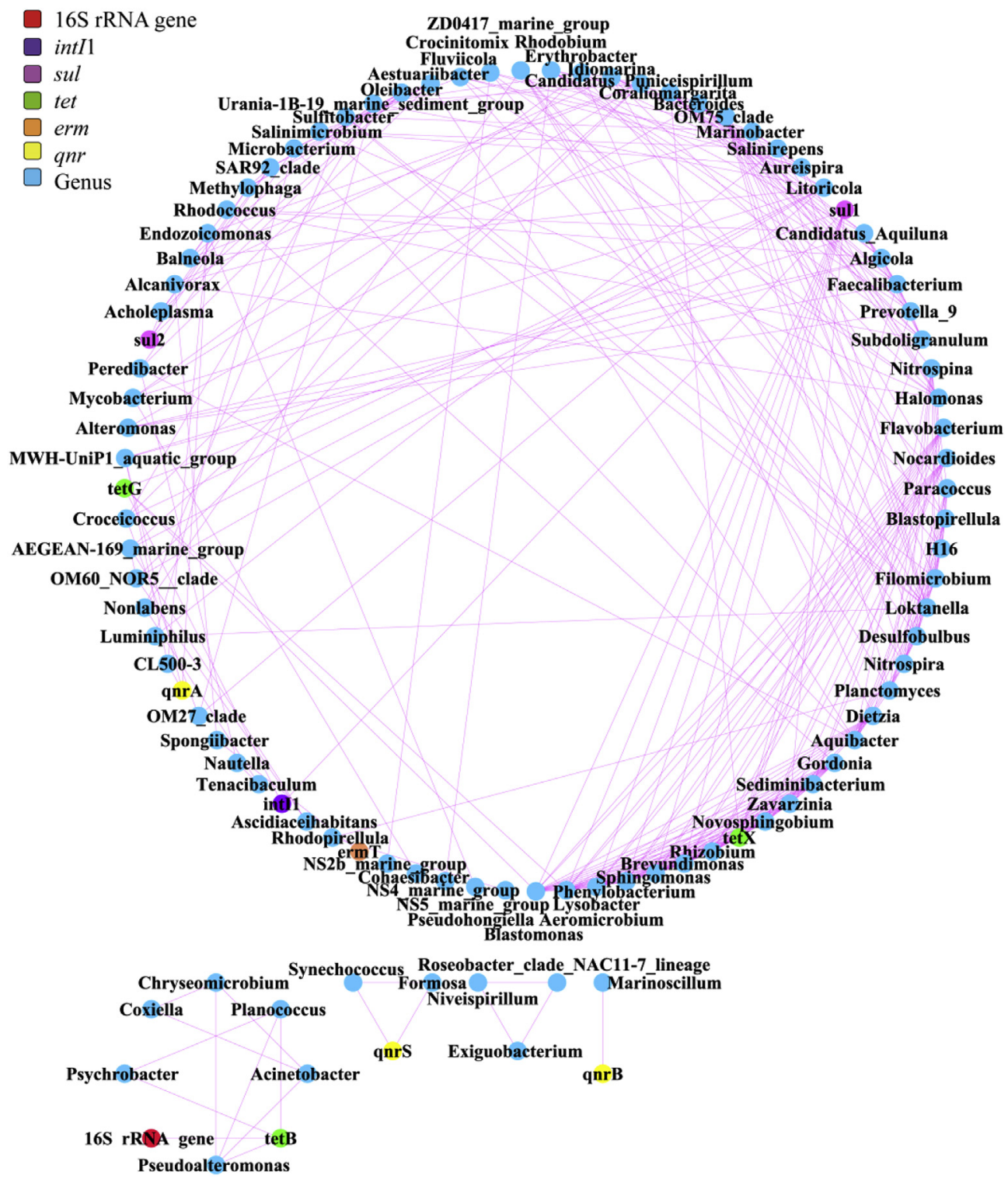

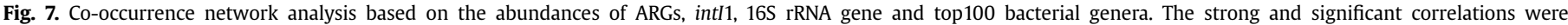

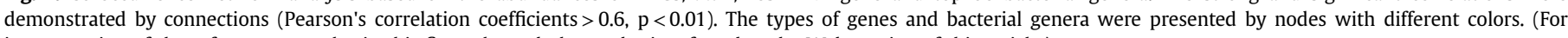
interpretation of the references to color in this figure legend, the reader is referred to the Web version of this article.) 
abundances more than $1.0 \%$ (Fig. S3).

To reveal which bacterial genera were carrying the antibiotic resistance genes and intI1, the network analysis was utilized to establish the co-occurrence patterns between ARGs, intI1, 16S rRNA gene and the top 100 bacterial genera based on the Pearson's correlations. As shown in Fig. 7, 321 pairs of significant and strong correlations $(\rho>0.6, p<0.01)$ were identified involving in 9 ARG subtypes, intI1, 16S rRNA gene and 90 bacterial genera. The correlations included $1.25 \%$ of Gene-Gene, $11.53 \%$ of ARG-Genus and $87.23 \%$ of Genus-Genus. It has been reported that the co-occurring events between bacterial species and ARGs were due to hosting relationship (Ju et al., 2016). Among the 37 ARG-Genus co-occurring events, ARGs with tetracycline resistance (tet), sulfonamide resistance (sul), quinolone resistance (qnr) and macrolide resistance (erm) accounted for 51.35\%, 29.73\%, 16.22\% and $2.70 \%$, respectively.

Aeromicrobium and Blastomonas had strong correlations with tetracycline resistance genes (tet $\mathrm{G}$ and tetX) and sulfonamide resistance genes (sul1). Meanwhile, two ARG subtypes (tetX and sul1) were assigned to Brevundimonas, Lysobacter, Phenylobacterium, Sphingomonas and Zavarzinia. It could be speculated that these bacteria were potential hosts of tetracycline resistance genes (tet) and sulfonamide resistance genes (sul), resulting in the development of antibiotic resistant bacteria after undergoing the horizontal gene transfer and selection pressures. To further investigate the distribution of potential antibiotic resistant bacteria carrying multiple ARGs, a contrast was made between abovementioned bacteria detected from the Yellow Sea with those from the Bohai Sea. Interestingly, Brevundimonas, Aeromicrobium, Zavarzinia, Blastomonas, Sphingomonas, Phenylobacterium and Lysobacter were all detected with higher relative abundances in the Yellow Sea than those in the Bohai Sea, indicating that the abundance of multiple antibiotic resistant bacteria in the Yellow Sea was higher than that in the Bohai Sea.

All these bacterial genera hold the highest abundances in YS01 among all the sampling sites in the Yellow Sea area. For example, the relative abundances of Brevundimonas, Aeromicrobium, Zavarzinia were up to $5.31 \%, 3.07 \%$ and $3.69 \%$, respectively. Notably, this result was in accordance with the quantification result of ARGs that the absolute abundances of sul $1\left(2.13 \times 10^{3}\right.$ copies $\left./ \mathrm{mL}\right)$, tet $\mathrm{G}(80.5$ copies $/ \mathrm{mL})$ and tetX $\left(1.68 \times 10^{3}\right.$ copies $\left./ \mathrm{mL}\right)$ were the highest detected in YS01 among all the seawater samples. YS01 was adjacent to the semi-enclosed bay (Jiaozhou Bay), in which the high concentrations of antibiotics were caused by the freshwater discharge and poor water exchange ability (Zhang et al., 2013). Consequently, the abundance of potential multiple antibiotic resistant bacteria topped at the sampling site near the southern of Shandong Peninsula.

\section{Conclusions}

The findings provided a comprehensive and new insight on the pollution status of ARGs in the Bohai Sea and Yellow Sea areas. ARGs were more abundant in coastal zone and rivers than in the Bohai Sea and Yellow Sea. The abundances of selected ARGs in the inshore areas were higher than those in the offshore areas and the abundances of ARGs in the inner bay were higher than those in the bay mouth. Owing to the complicated geographical environment and land-based discharge, ARGs fluctuated greatly in the Yellow Sea and in the coastal areas. Sulfonamide resistance genes hold the highest abundances in the sea, followed by tetracycline resistance genes (tet) and quinolone resistance genes (qnr), while the abundance of qnrA was the highest in the coastal zone and rivers. The distribution coefficients of target genes between sediment phase and water phase were more than 1.0 in the majority of selected aquatic systems, indicating that ARGs were inclined to be accumulated in sediments. Ammonium and nitrate played important roles in the distribution and variation of target genes. Water samples collected from the Bohai and Yellow Sea clustered together while samples from the coastal areas were separated, confirming that ARGs in aquatic systems along the coastline were influenced by complicated environmental factors in coastal areas. Co-occurrence network analysis revealed the potential multi-antibiotic resistant bacteria, which were detected with higher abundances in the Yellow Sea, and topped at the sampling site adjacent to the Jiaozhou Bay.

\section{Conflict of interest}

The authors declare no conflict of interest.

\section{Acknowledgements}

This work was supported by National Natural Science Foundation of China (Grant number of 41877131), Taishan Scholar Program of Shandong Province (No. tsqn201812116), One Hundred Talents Program of Chinese Academy of Sciences (Grant number of Y629041021), and Two-Hundred Talents Plan of Yantai (No. Y739011021).

\section{Appendix A. Supplementary data}

Supplementary data to this article can be found online at https://doi.org/10.1016/j.envpol.2019.05.143.

\section{References}

Cambau, E., Lascols, C., Sougakoff, W., Bébéar, C., Bonnet, R., Cavallo, J.D. Gutmann, L., Ploy, M.C., Jarlier, V., Soussy, C.J., Robert, J., 2006. Occurrence of qnrA-positive clinical isolates in French teaching hospitals during 2002-2005. Clin. Microbiol. Infect. 12, 1013-1020.

Chen, H.W., Lin, H.C., Chuang, Y.H., Sun, C.T., Chen, W.Y., Kao, C.Y., 2019a. Effects of environmental factors on benthic species in a coastal wetland by redundancy analysis. Ocean Coast Manag. 169, 37-49.

Chen, J., Su, Z., Dai, T., Huang, B., Mu, Q., Zhang, Y., Wen, D., 2019b. Occurrence and distribution of antibiotic resistance genes in the sediments of the East China Sea bays. J. Environ. Sci. 81, 156-167.

Dang, B., Mao, D., Xu, Y., Luo, Y., 2017. Conjugative multi-resistant plasmids in Haihe River and their impacts on the abundance and spatial distribution of antibiotic resistance genes. Water Res. 111, 81-91.

Di Cesare, A., Eckert, E.M., D'Urso, S., Bertoni, R., Gillan, D.C., Wattiez, R., Corno, G., 2016. Co-occurrence of integrase 1 , antibiotic and heavy metal resistance genes in municipal wastewater treatment plants. Water Res. 94, 208-214.

Duan, L., Song, J., Xu, Y., Li, X., Zhang, Y., 2010. The distribution, enrichment and source of potential harmful elements in surface sediments of Bohai Bay, North China. J. Hazard Mater. 183, 155-164.

Gao, Q., Li, Y., Qi, Z., Yue, Y., Min, M., Peng, S., Shi, Z., Gao, Y., 2018. Diverse and abundant antibiotic resistance genes from mariculture sites of China's coastline. Sci. Total Environ. 630, 117-125.

Ju, F., Li, B., Ma, L., Wang, Y., Huang, D., Zhang, T., 2016. Antibiotic resistance genes and human bacterial pathogens: Co-occurrence, removal, and enrichment in municipal sewage sludge digesters. Water Res. 91, 1-10.

Liu, X., Zhang, G., Liu, Y., Lu, S., Oin, P., Guo, X., Bi, B., Wang, L., Xi, B., Wu, F., Wang, W., Zhang, T., 2019. Occurrence and fate of antibiotics and antibiotic resistance genes in typical urban water of Beijing, China. Environ. Pollut. 246, $163-173$.

Lu, J., Wu, J., Zhang, C., Zhang, Y., Lin, Y., Luo, Y., 2018. Occurrence, distribution, and ecological-health risks of selected antibiotics in coastal waters along the coastline of China. Sci. Total Environ. 644, 1469-1476.

Lu, Z., Na, G., Gao, H., Wang, L., Bao, C., Yao, Z., 2015. Fate of sulfonamide resistance genes in estuary environment and effect of anthropogenic activities. Sci. Total Environ. 527-528, 429-438.

Luo, Y., Mao, D., Rysz, M., Zhou, Q., Zhang, H., Xu, L., J. J, Alvarez, P., 2010. Trends in antibiotic resistance genes occurrence in the Haihe River, China. Environ. Sci. Technol. 44, 7220-7225.

Ma, L., Li, B., Zhang, T., 2019. New insights into antibiotic resistome in drinking water and management perspectives: a metagenomic based study of smallsized microbes. Water Res. 152, 191-201.

Pruden, A., 2014. Balancing water sustainability and public health goals in the face of growing concerns about antibiotic resistance. Environ. Sci. Technol. 48, 5-14. 
Song, J., Duan, L., 2019a. Chapter 17 - the Bohai Sea. In: Sheppard, C. (Ed.), World Seas: an Environmental Evaluation, second ed. Academic Press, pp. 377-394.

Song, J., Duan, L., 2019b. Chapter 18 - the Yellow sea. In: Sheppard, C. (Ed.), World Seas: an Environmental Evaluation, second ed. Academic Press, pp. 395-413.

Su, H., Liu, S., Hu, X., Xu, X., Xu, W., Xu, Y., Li, Z., Wen, G., Liu, Y., Cao, Y., 2017. Occurrence and temporal variation of antibiotic resistance genes (ARGs) in shrimp aquaculture: ARGs dissemination from farming source to reared organisms. Sci. Total Environ. 607-608, 357-366.

Subirats, J., Timoner, X., Sànchez-Melsió, A., Balcázar, J.L., Acuña, V., Sabater, S., Borrego, C.M., 2018. Emerging contaminants and nutrients synergistically affect the spread of class 1 integron-integrase (intl1) and sul1 genes within stable streambed bacterial communities. Water Res. 138, 77-85.

Wang, J.-H., Lu, J., Wu, J., Zhang, Y., Zhang, C., 2019. Proliferation of antibiotic resistance genes in coastal recirculating mariculture system. Environ. Pollut. $248,462-470$
Zhang, Q.Q., Ying, G.G., Pan, C.G., Liu, Y.S., Zhao, J.L., 2015. Comprehensive evaluation of antibiotics emission and fate in the river basins of China: source analysis, multimedia modeling, and linkage to bacterial resistance. Environ. Sci. Technol. 49, 6772-6782.

Zhang, R., Tang, J., Li, J., Cheng, Z., Chaemfa, C., Liu, D., Zheng, Q., Song, M., Luo, C. Zhang, G., 2013. Occurrence and risks of antibiotics in the coastal aquatic environment of the Yellow Sea, North China. Sci. Total Environ. 450-451, 197-204.

Zhang, Y., Niu, Z., Zhang, Y., Zhang, K., 2018. Occurrence of intracellular and extracellular antibiotic resistance genes in coastal areas of Bohai Bay (China) and the factors affecting them. Environ. Pollut. 236, 126-136.

Zhu, Y.-G., Zhao, Y., Li, B., Huang, C.-L., Zhang, S.-Y., Yu, S., Chen, Y.-S., Zhang, T. Gillings, M.R., Su, J.-Q., 2017. Continental-scale pollution of estuaries with antibiotic resistance genes. Nature Microbiol. 2, 16270. 